\title{
Comparison of various adiposity indexes in women with polycystic ovary syndrome and normo-ovulatory non-hirsute women: a population-based study
}

\author{
Fahimeh Ramezani Tehrani ${ }^{1}$, Sonia Minooee ${ }^{1}$ and Fereidoun Azizi ${ }^{2}$ \\ ${ }^{1}$ Reproductive Endocrinology Research Center and ${ }^{2}$ Endocrine Research Center, Research Institute for \\ Endocrine Sciences, Shahid Beheshti University of Medical Sciences, 24 Parvaneh, Yaman Street, Velenjak, \\ PO Box 19395-4763, Tehran, Iran
}

Correspondence should be addressed to F Ramezani Tehrani Email

ramezani@endocrine.ac.ir

\begin{abstract}
Objective: Insulin resistance (IR) and metabolic disorders are common in polycystic ovary syndrome (PCOS). However, it is still not clear which adiposity marker could precisely predict metabolic syndrome (MetS) in women with PCOS and whether these indexes are different in normo-ovulatory non-hirsute women.

Design: A case-control study was conducted on a total of 175 Iranian subjects with PCOS and 525 normal control subjects, aged 18-45 years.

Methods: Waist circumference (WC), BMI, waist-to-hip ratio, lipid accumulation product (LAP) index, and visceral adiposity index (VAI) were examined and the homeostasis model assessment index was calculated. MetS was defined according to the joint interim statement. The receiver operating characteristic curves were used to evaluate the extent to which measures of adiposity can predict IR and MetS risk.

Results: LAP index and VAl are two indicators (sensitivity and PPV of 70\% (LAP index) and $60 \%$ (VAI), and $80 \%$ (LAP index) and $83 \%$ (VAI) respectively) that best predict IR in women with PCOS. Among healthy women, the LAP index and WC were better markers (sensitivity and PPV of 78\% (LAP index) and 75\% (VAI), and 82\% (LAP index) and 81\% (VAI) respectively). The two most reliable indicators for prediction of MetS among PCOS and normal women were the WC and VAI (sensitivity and PPV of $83 \%$ (WC) and $81 \%$ (VAI), and $97 \%$ (WC) and 95\% (VAI) respectively) and the VAI and LAP index (sensitivity and PPV of $88 \%$ (VAI) and $83 \%$ (LAP index), and 98\% (VAI) and 98\% (LAP index) respectively) respectively.

Conclusions: While the appropriate adiposity indicators and their optimum cutoff values vary in women with PCOS, compared with the normal control subjects, the LAP index is an easily obtainable index that might be useful for screening of cardiometabolic complications among both groups.
\end{abstract}

European Journal of

Endocrinology

(2014) 171, 199-207

\section{Introduction}

Polycystic ovary syndrome (PCOS), a disorder characterized by ovulatory dysfunction and hyperandrogenism (HA), is the most prevalent endocrinopathy in women of reproductive age. PCOS is also considered a metabolic disorder, as insulin resistance (IR), an independent risk factor for cardiovascular disease (CVD), and central body fat accumulation independent of obesity are the common features in these patients (1).

(C) 2014 European Society of Endocrinology Printed in Great Britain
While there is controversy on the association between PCOS and metabolic syndrome (MetS) (2), the Androgen Excess Society in a recent consensus statement has, however, highlighted the primary importance of prevention of cardiometabolic complications in women with PCOS and has recommended determining the BMI, waist circumference (WC), serum lipid/glucose, and blood pressure in all women with PCOS (3). It has also been

Published by Bioscientifica Ltd. 
demonstrated that IR is increased in women with PCOS (4), which could possibly expose these women to further metabolic disturbances $(5,6)$.

There is uncertainty regarding the association between PCOS and obesity, and it seems that in an unselected population this association might be minimal (7). Furthermore, it is still not clear as to which of the various simple and complex adiposity indexes could precisely predict the metabolic disturbances $(8,9,10)$ and whether these indexes are different in women with PCOS compared with normoovulatory non-hirsute women (8). It has been shown that the BMI cannot be efficiently used to precisely predict CVD risk $(11,12)$. In comparison with the BMI, other indicators of central adiposity, such as the WC, have more capacity to predict obesity-related cardiovascular risk and have been used as a criterion for clinical diagnosis of MetS (13). Although the WC has been reported to be more closely correlated with the amount of abdominal fat in comparison with the waist-to-hip ratio (WHR) (14), the WHR is also widely used to investigate the relations between abdominal fat and metabolic profiles (15). Several other complex indicators have been reported for better assessment of cardiometabolic risks, with various results $(16,17)$.

Although there are some studies that reported a similarity between cardiovascular risk assessments in all reproductiveage women, regardless of their PCOS status (18), others demonstrated a difference in the metabolic risk assessment of women with PCOS compared with their normo-ovulatory counterparts $(6,19)$. In a study by Amato et al. (9), the visceral adiposity index (VAI) was introduced as a measure associated with cardiovascular risk in both normal subjects and those with PCOS, and Wiltgen et al. (20) reported the lipid accumulation product (LAP) index as an accepted discriminator of IR among women with PCOS and healthy women.

Considering the lack of population-based studies and the lack of consensus on the best predictor of cardiometabolic risks in women with PCOS in comparison to normal populations, this study aimed to compare the validity of various available indicators on data derived from a geographically diverse group of women with PCOS - participants of a large population-based study.

\section{Subjects and methods}

This study was approved by the ethical review board of the Research Institute for Endocrine Sciences and informed consent was obtained from all participants. Subjects of this study included individuals from two large populationbased studies conducted in five provinces of different geographic regions of Iran, the first study (21) including
1126 women aged 18-45 years living in urban areas of Ghazvin, Kermanshah, Golestan, and Hormozgan, and the second study included 646 reproductive-age women (18-45 years) living in urban areas of Khuzestan province (22); study protocols and the main investigator (F R T) were the same for both of them. Menopausal women, those who had undergone hysterectomy or bilateral oophorectomy, and pregnant women were excluded. We further excluded those women who had hyperprolactinemia and thyroid dysfunction, women with only androgen excess, women with only ovulatory dysfunction, and women with only polycystic ovaries (PCOs). Finally, the remaining participants $(n=700)$ were divided into two study groups as follows: PCOS $(n=175)$ by Rotterdam criteria (23) and healthy control subjects $(n=525)$. The details of the study protocol have been published previously (21).

\section{Assays}

All participants underwent clinical examinations, where body weight, height, waist and hip circumferences, and blood pressure were measured by the trained staff. Height and weight were measured with subjects in light clothes and without shoes, using a standard apparatus. Weight was measured to the nearest $0.1 \mathrm{~kg}$ on a calibrated beam scale. The height and WC were measured to the nearest $0.5 \mathrm{~cm}$ with a measuring tape. Waist was measured midway between the lower rib margin and the iliac crest at the end of a gentle expiration. BMI was calculated as the weight in kilograms divided by the height in meters squared $\left(\mathrm{kg} / \mathrm{m}^{2}\right)$.

For biochemical and hormonal measurements, overnight fasting blood samples after $12 \mathrm{~h}$ were taken from each subject on the second or third day of their spontaneous or progesterone-induced menstrual cycles. Blood samples were collected in EDTA-treated test tubes. Plasma was separated in a refrigerated centrifuge at $1560 \boldsymbol{g}$ for $10 \mathrm{~min}$ and sera were stored at $-80^{\circ} \mathrm{C}$ until tested. Fasting plasma glucose (FPG) was measured using an enzymatic colorimetric method with glucose oxidase.

Lipid measurements including total cholesterol (TC), triglycerides (TGs), and HDL-C were obtained using commercial assay kits (Pars Azmoon, Inc., Tehran, Iran). TC and TGs were assayed using enzymatic colorimetric tests with cholesterol esterase and cholesterol oxidase, and glycerol phosphate oxidase respectively. HDL-C was measured after precipitation of the apolipoprotein $B$ containing lipoproteins with phosphotungstic acid. All samples were analyzed when internal quality control met the acceptable criteria. Insulin was assessed by ImmunoEnzyme Metric Assay (IEMA; Mercodia, Uppsala, Sweden). 
DHEAS and total testosterone (TT) were measured by enzyme immune assay (Diagnostic Biochem Canada Co., ON, Canada). Sex hormone-binding globulin (SHBG) was measured by IEMA (Diagnostic Biochem Canada Co.). All ELISAs were performed using a Sunrise ELISA reader (Tecan Co., Salzburg, Austria). Leutinizing hormone (LH) and follicle-stimulating hormone (FSH) were measured by IRMA (Izotop, Budapest, Hungary) using a gamma counter (Wallac Wizard, Turku, Finland). The free androgen index (FAI) was calculated using the formula (TT $(\mathrm{nmol} / \mathrm{l}) \times$ 100/SHBG (nmol/l)). The intra- and inter-assay coefficient of variation (CV) values were 2.5 and $3.2 \%$ for TC, 2.2 and $2.3 \%$ for TGs, 5.6 and $6.6 \%$ for $\mathrm{TT}, 5.1$ and $6.8 \%$ for DHEAS, 1.2 and $5.7 \%$ for SHBG, 3 and $5.8 \%$ for $\mathrm{LH}$, and 3.5 and $4.0 \%$ for $\mathrm{FSH}$ respectively.

All of the ever married study subjects (82\%) were invited for transvaginal ultrasound scans and never married (18\%) for transabdominal ultrasound scans of the ovaries, which were performed using the $3.5-\mathrm{MHz}$ transabdominal and $5-\mathrm{MHz}$ transvaginal transducer by an experienced sonographer in each province and all scans were assessed by a single sonographer. Ultrasound was performed on the same day as the blood samples were collected.

\section{Definitions}

MetS was defined, according to the joint interim statement (JIS) (24), as the presence of any three of the following five risk factors: i) abdominal obesity defined by a WC of $91 \mathrm{~cm}$ according to the population and country-specific cutoff point for Iranian women (25); ii) FPG of $100 \mathrm{mg} / \mathrm{dl}$ or drug treatment; iii) fasting TGs of $150 \mathrm{mg} / \mathrm{dl}$ or drug treatment; iv) fasting HDL-C $<50 \mathrm{mg} / \mathrm{dl}$ in women or drug treatment; and $\mathrm{v}$ ) raised blood pressure defined as systolic blood pressure (SBP) of $130 \mathrm{mmHg}$, diastolic blood pressure (DBP) of $85 \mathrm{mmHg}$, or antihypertensive drug treatment.

The biochemical hyperandrogenemia was defined using the upper 95th percentile for the normal women who were not on any hormonal medication and had no clinical evidence of $\mathrm{HA}$, oligo/anovulation (ANOVU), or PCOs. These values were 5.47 and $0.88 \mathrm{ng} / \mathrm{ml}$ for the FAI and TT respectively.

In this study, PCOS was defined according to the Rotterdam (Rott) criteria as the presence of two or more of the following risk factors: oligo/amenorrhea, clinical and/or biochemical HA, and PCOs (23). Brief description of each of these criteria has been reported previously $(21,26)$. These women with PCOS were further subdivided into three sub

Table 1 Clinical, hormonal, and metabolic features of normal participants and various phenotypes of PCOS using the Rotterdam criteria. Values are given as median (inter quartile range).

\begin{tabular}{|c|c|c|c|c|}
\hline & $\begin{array}{c}\text { Group } 1(n=80) \text { ANOVU }+ \text { HA } \\
\text { or full Rotterdam criteria }\end{array}$ & Group $2(n=65)$ PCO + HA & $\begin{array}{c}\text { Group } 3(n=30) \\
\text { ANOVU }+ \text { PCO }\end{array}$ & Normal $(n=525)$ \\
\hline Age (years) & *30.6 (28.2-31.8) & §29.2 (26.7-31.7) & *34.5 (31.7-37.3) & 27.6 (27-28.3) \\
\hline BMI $\left(\mathrm{kg} / \mathrm{m}^{2}\right)$ & $26.5(25.3-27.7)$ & $24.6(22.8-26.5)$ & $26.6(24.8-28.5)$ & $24.7(24.2-25.2)$ \\
\hline WHR & $0.81(0.79-0.83)$ & $0.78(0.75-0.8)$ & $0.8(0.79-0.84)$ & $0.80(79-0.81)$ \\
\hline WC $(\mathrm{cm})$ & $81.7(79.1-84.3)$ & §80.2 (75.9-84.6) & $*^{*} \neq 86.8(82-91.6)$ & $78.3(77.2-79.4)$ \\
\hline SBP $(\mathrm{mmHg})$ & 106.8 (104.3-109.3) & $106.2(101.6-110.9)$ & $107.04(103.1-110.9)$ & 109.9 (107.3-112.6) \\
\hline DBP (mmHg) & $68.1(65.7-70.5)$ & $66.4(62.5-70.2)$ & $66.8(63.1-70.5)$ & $69.3(67.6-71.1)$ \\
\hline FPG (mmol//l) & $4.8(4.7-5)$ & $4.6(4.3-4.9)$ & $4.9(4.4-5.4)$ & $5(4.9-5.2)$ \\
\hline $\mathrm{TG}(\mathrm{mmol} / \mathrm{l})$ & $1.3(1.1-1.5)$ & $1.41(1.1-1.7)$ & $1.4(1.19-1.69)$ & $1.2(1.1-1.3)$ \\
\hline $\mathrm{TC}(\mathrm{mmol} / \mathrm{l})$ & $4.2(4-4.3)$ & $4.3(4-4.5)$ & $4.3(4-4.6)$ & $4.1(4-4.2)$ \\
\hline HDL-C (mmol/l) & $1.1(1.09-1.23)$ & $1.2(1.08-1.3)$ & $1.1(1.05-1.2)$ & $1.2(1.1-1.2)$ \\
\hline LDL-C (mmol/l) & $2.5(2.3-2.6)$ & $2.4(2.2-2.7)$ & $2.6(2.3-2.8)$ & $2.3(2.3-2.4)$ \\
\hline LH (IU/I) & $6.1(5.1-7.1)$ & $6.2(4.2-8.1)$ & $5.07(3.9-6.2)$ & $6(5.4-6.6)$ \\
\hline SHBG (nmol/l) & *51.4 (45.3-57.5) & $60.1(51.2-69)$ & ${ }^{ \pm} 69.3(59.1-79.4)$ & $64.2(60.3-68.1)$ \\
\hline $\mathrm{FAI}$ & *3.5 (2.6-4.4) & *5.8 (5.02-6.7) & ${ }^{\ddagger}+{ }^{+} 2.27(1.8-2.7)$ & $1.1(1.05-1.3)$ \\
\hline TT (nmol/l) & *0.68 (0.6-0.7) & *0.94 (0.7-1.1) & ${ }^{\ddagger}+{ }^{\dagger} 0.4(0.3-0.4)$ & $0.46(0.43-0.49)$ \\
\hline HOMA-IR index & $2.2(1.7-2.7)$ & $1.9(1.5-2.3)$ & $1.7(1.3-2.2)$ & $2.1(1.8-2.3)$ \\
\hline VAl & $2.6(1.8-3.4)$ & $2.6(1.6-3.6)$ & $2.5(1.9-3.1)$ & $2.3(1.9-2.7)$ \\
\hline LAP index & *, $35.2(28.1-42.3)$ & ${ }^{*}+{ }^{+\dagger} 37.01(22.9-51.1)$ & *43.3 (31.5-55.1) & $28.9(25.9-31.8)$ \\
\hline IR (\%) & *32 (40) & *22 (33) & *11(36) & 99 (19) \\
\hline MetS $^{a}(\%)$ & *14 (17) & $12(18)$ & $5(16)$ & $52(10)$ \\
\hline
\end{tabular}

PCOS, polycystic ovary syndrome; WHR, waist-to-hip ratio; WC, waist circumference; SBP, systolic blood pressure; DBP, diastolic blood pressure; FPG, fasting plasma glucose; TGs, triglycerides; TC, total cholesterol; LH, luteinizing hormone; SHBG, sex hormone-binding globulin; FAI, free androgen index; TT, total testosterone; HOMA-IR, homeostasis model assessment-insulin resistance; VAI, visceral adiposity index; LAP, lipid accumulation product; IR, insulin resistance; MetS, metabolic syndrome; ANOVU, anovulation; PCO, polycystic ovary; HA, hyperandrogenemia. ${ }^{\star V}$ s normal group, $P<0.05 ;{ }^{\dagger}$, Group 1 vs Group 2, $P<0.05 ;{ }^{\ddagger}$, Group 1 vs Group 3; ${ }^{\S}$, Group 2 vs Group $3, P<0.05 .{ }^{\dagger+}, P<0.05 ;{ }^{\dagger}, P<0.05 ; P<0.05$ : Group 1 vs Group 3.

${ }^{a}$ According to JIS criteria. 
groups as: i) ANOVU + hyperandrogenemia and/or HA with or without PCOs, ii) ANOVU+ PCO, and iii) HA+PCO.

IR was estimated by the homeostasis model assessment (HOMA-IR) as a surrogate for the measurement of IR according to the following formula (27): HOMA-IR= ((fasting insulin level $(\mathrm{mU} / \mathrm{l}) \times(\mathrm{FPG} \quad(\mathrm{mmol} / \mathrm{l})) / 22.5$. To determine the IR cutoff value, 129 subjects with a BMI $<25 \mathrm{~kg} / \mathrm{m}^{2}$, non-diabetic, non-hypertensive (fasting blood sugar (FBS) <126 (mg/dl); BP=130/85 (mmHg)), and without any metabolic abnormalities, hirsutism, or PCOs (in ultrasonography) were selected; in these participants, the HOMA-IR distribution had some skewness and 90th percentile was considered for the definition of IR $($ HOMA-IR $=2.3)(28)$.

The LAP index was calculated using the formula (waist $(\mathrm{cm})-58) \times \mathrm{TG}$ concentration $(\mathrm{mmol} / \mathrm{l})$, as reported by Kahn (2005) (16).

The VAI was calculated, as described by Amato et al. (2010) (17), using the following sex-specific equation:

where TG concentration and HDL concentration were expressed in $\mathrm{mmol} / \mathrm{l}$.

\section{Statistical analysis}

The Statistical Package for Social Sciences SPSS version 21 was used for data analysis. Baseline characteristics are expressed as the mean \pm s.D. and median (IQ 25-75) as appropriate. The normality of distribution for quantitative data was assessed by the Kolmogorov-Smirnov test. Differences between groups were compared by one-way ANOVA test, followed by post hoc test for multiple comparisons (after testing for the equality of variance using the Levene test).

The receiver operating characteristic (ROC) curves were generated for five previously reported adiposity indicators $(24,25)$. Sensitivity (SS), specificity (SP), area under curve (AUC), and positive and negative predictive values (PPV and NPV) were calculated for the markers. The optimal values for SS and SP were identified as the ones that keep $(1-S S)^{2}+(1-S P)^{2}$ at the minimum (29) and the Youden index at the maximum (30). The value of $P<0.05$ was considered statistically significant.

\section{Results}

In this study, women with PCOS were older $(31 \pm 7.6$ vs $27.6 \pm 5.5$ years), had a higher BMI $(26.1 \pm 5.1$ vs

$\left.24.7 \pm 4.6 \mathrm{~kg} / \mathrm{m}^{2}\right)$, and had a greater WC $(83.1 \pm 11.8$ vs $78.3 \pm 10.9 \mathrm{~cm})$ than control subjects $(P<0.001)$. The demographic, anthropometric, and metabolic features of study subjects including 175 with PCOS, which were
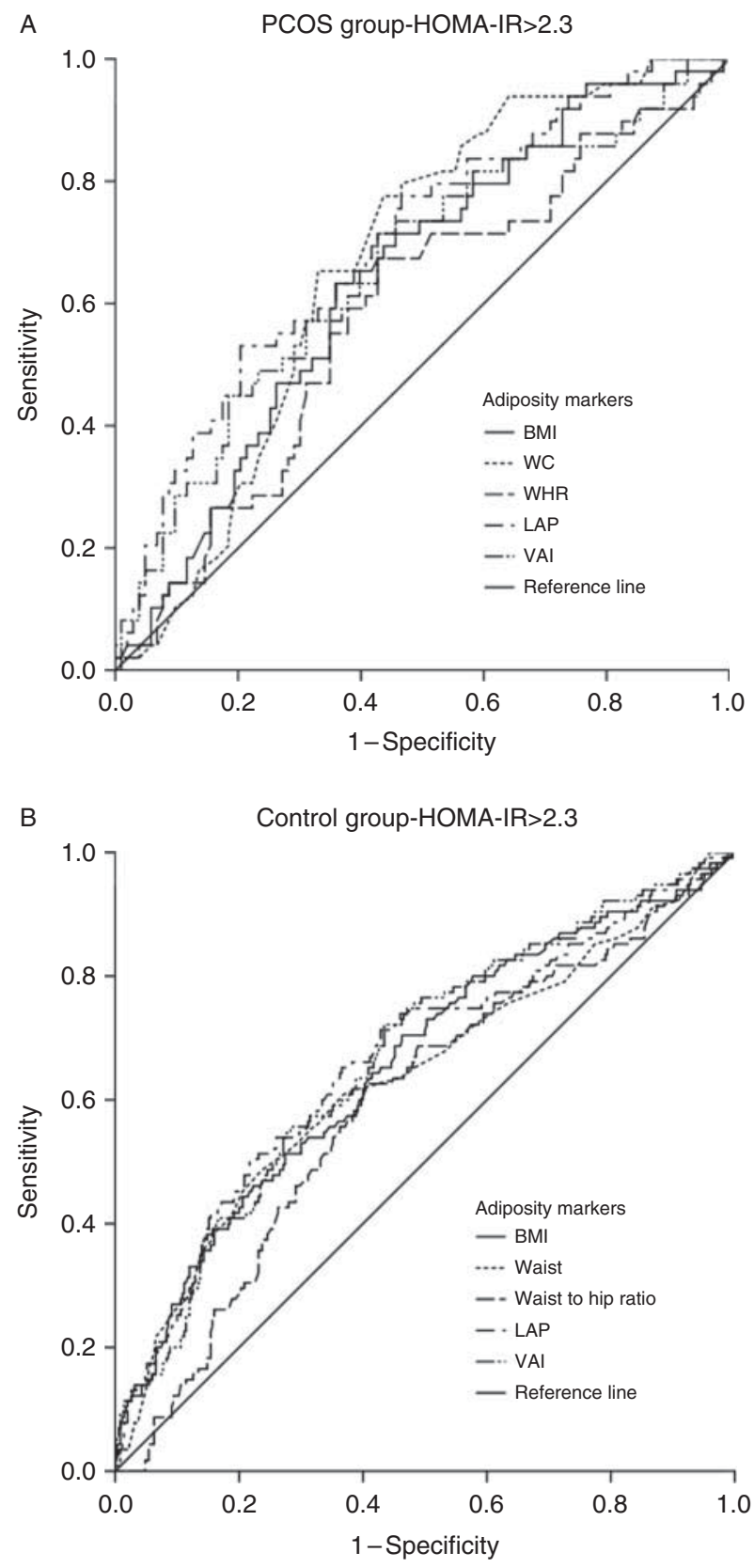

\section{Figure 1}

ROC curves of adiposity indexes in insulin resistance prediction (A-case and B-control groups). PCOS, polycystic ovary syndrome; HOMA-IR, homeostasis model assessment-insulin resistance; WHR, waist-to-hip ratio; WC, waist circumference; VAl, visceral adiposity index; LAP, lipid accumulation product. 
Table 2 Comparison of adiposity indexes that best predict insulin resistance (HOMA index $>2.3$ ).

\begin{tabular}{l} 
Adiposity \\
indexes cutoff \\
values \\
\hline BMI \\
WC \\
WHR \\
LAP \\
VAI
\end{tabular}

\begin{tabular}{rcc}
\multicolumn{2}{c}{ Cutoff values } \\
\hline PCOS & & Normal \\
\cline { 1 - 1 } 26.1 & & 25.6 \\
79.5 & & 83.5 \\
0.8 & & 0.79 \\
33.8 & & 34.7 \\
1.8 & & 1.6 \\
\hline
\end{tabular}

\begin{tabular}{|c|c|c|c|}
\hline \multicolumn{2}{|c|}{ AUC } & \multicolumn{2}{|c|}{ SS (\%) } \\
\hline PCOS & Normal & PCOS & Normal \\
\hline 0.63 & 0.61 & 0.61 & 0.7 \\
\hline 0.67 & 0.61 & 0.57 & 0.75 \\
\hline 0.62 & 0.59 & 0.57 & 0.53 \\
\hline 0.65 & 0.64 & 0.7 & 0.78 \\
\hline 0.66 & 0.63 & 0.6 & 0.56 \\
\hline
\end{tabular}

\begin{tabular}{|c|c|}
\hline \multicolumn{2}{|c|}{ SP (\%) } \\
\hline PCOS & Normal \\
\hline 0.66 & 0.52 \\
\hline 0.78 & 0.47 \\
\hline 0.67 & 0.64 \\
\hline 0.6 & 0.51 \\
\hline 0.72 & 0.7 \\
\hline
\end{tabular}

\begin{tabular}{|c|c|c|c|}
\hline \multicolumn{2}{|c|}{ PPV (\%) } & \multicolumn{2}{|c|}{ NPV (\%) } \\
\hline PCOS & Normal & PCOS & Normal \\
\hline 0.8 & 0.81 & 0.42 & 0.37 \\
\hline 0.85 & 0.81 & 0.44 & 0.39 \\
\hline 0.78 & 0.82 & 0.42 & 0.31 \\
\hline 0.8 & 0.82 & 0.46 & 0.44 \\
\hline 0.83 & 0.85 & 0.43 & 0.35 \\
\hline
\end{tabular}

PCOS, polycystic ovary syndrome; WHR, waist-to-hip ratio; WC, waist circumference; VAI, visceral adiposity index; LAP, lipid accumulation product; HOMA homeostasis model assessment; AUC, area under curve; SS, sensitivity; SP, specificity; PPV, positive predictive value; NPV, negative predictive value.

subdivided into three phenotypes, and 525 normal control subjects are presented in Table 1. Except the ANOVU+ $\mathrm{HA} \pm$ PCO subgroup, subjects with PCOS had no statistically significant difference in lipid and glucose profiles in comparison with the normal control subjects. Although MetS was more prevalent in women with PCOS, it was not statistically significant (Table 1 ). The prevalence of IR in $\mathrm{ANOVU}+\mathrm{HA} \pm \mathrm{PCO}, \mathrm{HA}+\mathrm{PCO}$, and $\mathrm{ANOVU}+\mathrm{PCO}$ were 40,33 , and $36 \%$ respectively; they were significantly more than that observed in normal women $(19 \%, P<0.001$; Table 1).

In women with PCOS, there was a strong significant correlation between TG level with VAI and LAP index ( $P$ value: $0.001, r$ : 0.9 and $P$ value: $0.001, r: 0.8$ respectively); a high correlation was also observed between HDL-C level and VAI ( $P$ value: $0.001, r:-0.6)$. In control subjects, we observed a statistically significant correlation between TGs with VAI and LAP index ( $P$ value: $0.001, r: 0.8$ and $P$ value: $0.001, r$ : 0.8 respectively). The correlation between HDL-C level and VAI was also statistically significant ( $P$ value: $0.001, r:-0.5)$. Although the correlation between hormonal values and anthropometric or metabolic indexes was significant, their $\mathrm{CV}$ values were low (results not shown).

The ROC curve for participants with PCOS showed that the optimal cutoff values for different adiposity markers in predicting IR were as follows: BMI, $26.1 \mathrm{~kg} / \mathrm{m}^{2}$; WC, $79.5 \mathrm{~cm}$; WHR, 0.8; LAP index, 33.8; and VAI, 1.8 (Fig. 1A); these values for control women were $25.6 \mathrm{~kg} / \mathrm{m}^{2}, 83.5 \mathrm{~cm}, 0.79,34.7$, and 1.6 respectively (Fig. 1B). Table 2 demonstrated the SS, SP, PPV, NPV, and optimum value of different indexes for prediction of IR among women with PCOS and control women. Among these various indicators, the LAP index was the optimum marker with a SS of $70 \%$, SP of $60 \%$, PPV of $80 \%$, and NPV of $46 \%$ in women with PCOS. Comparing the ROC curves, we found that the WC and VAI had the most AUC $(0.67$ and 0.66 respectively) and LAP index had the highest SS
(0.7) in women with PCOS (Table 2). The results revealed that, in healthy control subjects, the LAP index and VAI were better predictors of IR (AUC of 0.64 and 0.63 respectively) and the LAP index and WC had the highest SS (0.78 and 0.75 respectively) (Table 2 ).

Figure 2A shows the ROC analysis of adiposity markers in predicting MetS in women with PCOS. The optimal cutoff values for different adiposity markers in predicting MetS were as follows: BMI, $27.1 \mathrm{~kg} / \mathrm{m}^{2}$; WC, $89.5 \mathrm{~cm}$; WHR, 0.84; LAP index, 38; and VAI, 3.1 in patients with PCOS; these values for control women were $27.1 \mathrm{~kg} / \mathrm{m}^{2}$, $88.5 \mathrm{~cm}, 0.78,36.9$, and 2.8 respectively (Fig. 2B). Among women with PCOS, we found that the WC and LAP index had the most AUC (0.85 and 0.83 respectively) and the WC and VAI had the highest SS (0.83 and 0.81 respectively) for prediction of MetS (Table 3 ). The results revealed that, in healthy control subjects, the VAI and LAP index were better predictors of MetS (AUC of 0.89 and 0.86 respectively) and the VAI and WC had the highest SS (0.88 and 0.87 respectively).

\section{Discussion}

In a large population-based study, we demonstrated that the LAP index $\geq 33.8$ and the VAI $\geq 1.8$ are two indicators (sensitivity and PPV of 70\% (LAP index) and 60\% (VAI), and $80 \%$ (LAP index) and 83\% (VAI) respectively) that best predict IR in women with PCOS. Among normoovulatory non-hirsute women, the two most reliable indicators were the LAP index and WC that had the optimum sensitivity of $78 \%$ and $75 \%$ and PPV of $82 \%$ and $81 \%$ respectively and were significantly correlated with our gold standard in cases with PCOS. The mean values of the WC and LAP index in women with PCOS that predict MetS was $89.5 \mathrm{~cm}$ and 38 respectively; they were more than those obtained in normal women (88.5 and 36.9 respectively). 
A
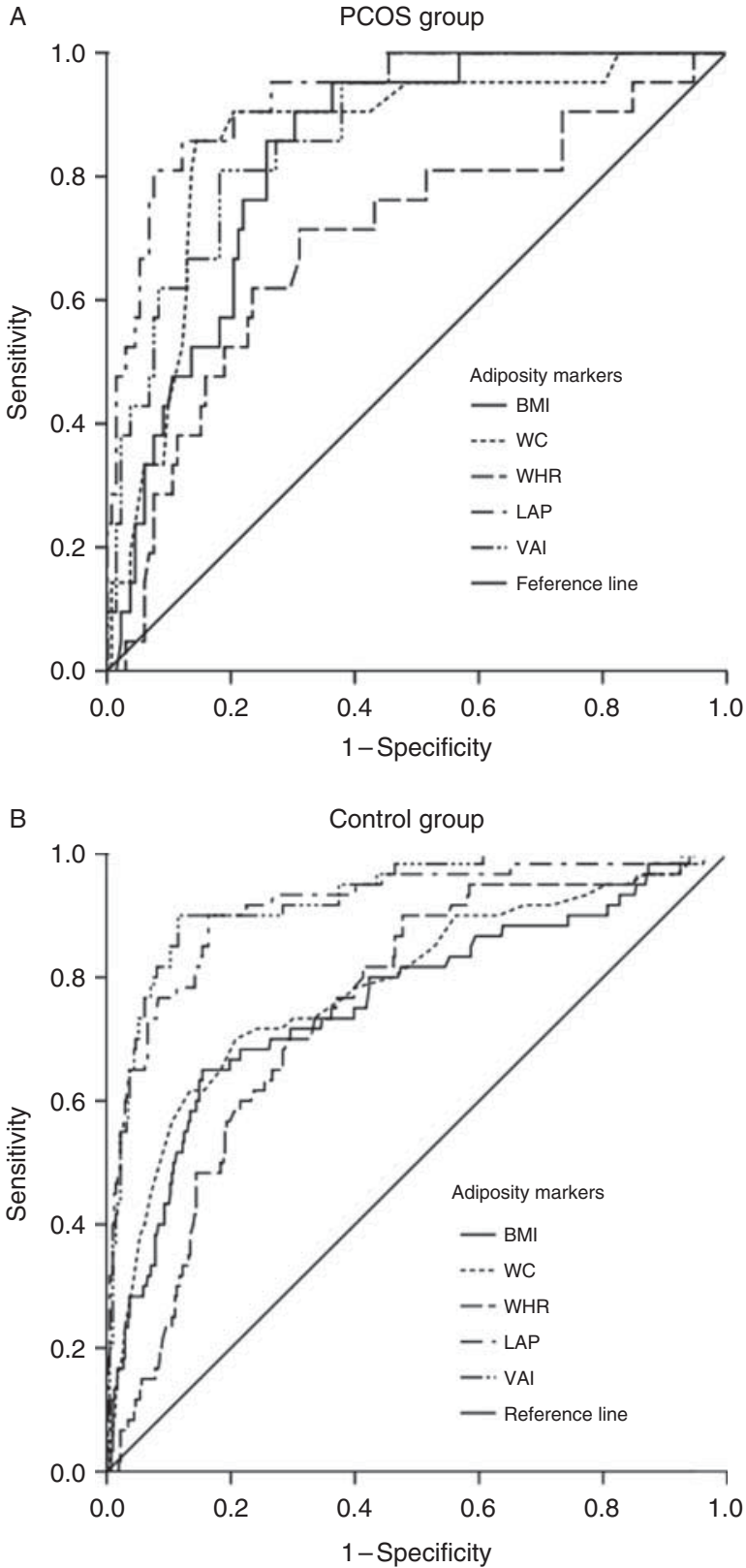

\section{Figure 2}

ROC curves of adiposity indexes in metabolic syndrome risk prediction (A-case and B-control groups). PCOS, polycystic ovary syndrome; HOMA-IR, homeostasis model assessment-insulin resistance; WHR, waist-to-hip ratio; WC, waist circumference; VAI, visceral adiposity index; LAP, lipid accumulation product.

The reported prevalence of obesity in women with PCOS is highly variable (31) and is limited by the lack of representative population-based data (32). Furthermore, it appears that the type of adiposity in women with PCOS varies from that of their normal counterparts $(33,34,35,36)$ and women with PCOS have a greater tendency to accumulate fat in the upper body when compared with control subjects matched for weight or BMI $(33,37)$; this effect could also be present even in lean women with PCOS $(33,38)$. Although lean women with PCOS are not necessarily more insulin resistant than healthy control subjects, a high BMI could exacerbate this condition in all women $(39,40,41)$.

It has been demonstrated that central adiposity is associated with more severe metabolic complications $(33,34,35)$ and several markers have been introduced for the precise assessment of adiposity. However, it is not clear whether there is any difference between the most appropriate adiposity indexes of women with PCOS and the normo-ovulatory non-hirsute women (8). Several efforts have been made to compare the optimum obesity marker for women with PCOS with normal control subjects $(8,9,10)$, but the majority of these studies are based on the measurements performed in women with PCOS referred to clinics $(42,43,44,45,46)$, which may not yield reliable estimations. In addition to small samples, these studies are restricted by the lack of comparison of various available adiposity markers. However, the population-based design of our study enables us to overcome the possible limitations of previous studies.

Several studies have been conducted to evaluate the prevalence of MetS in women with PCOS $(6,9,47)$; our results contrast with some other studies $(6,19)$ and demonstrated no significant increased prevalence of MetS in women with PCOS. It appears that in a population-based study on PCOS, the metabolic disturbances are not as severe as those observed in clinical or hospital-based studies, mainly due to the inclusion of those milder phenotypes of PCOS that are possibly not referred to the clinics. In this study, in agreement with other studies $(47,48)$, we observed that women with various PCOS phenotypes, especially those with more severe types (ANOVU $+\mathrm{HA} \pm$ PCO), had a higher IR prevalence than control subjects; however, this prevalence was much lower than in American women with PCOS (37 vs 64\%) (49).

Several pathogenesis are contributing to IR in PCOS (50). It has been shown that IR in women with PCOS may be due to androgen exposure at critical periods or has occurred because of intrauterine growth restriction (50); genetic disruption of insulin signaling in the brain is another mechanism that may have an important role in both ovulation and regulation of body weight (51).

Among the available adiposity complex indicators, the LAP index and VAI give promising results for the prediction of the cardiometabolic events in both normal women and those with PCOS $(9,20)$. The LAP index expresses both 
Table 3 Comparison of adiposity indexes that best predict MetS.

\begin{tabular}{l} 
Adiposity \\
indexes cutoff \\
values \\
\hline BMI \\
WC \\
WHR \\
LAP \\
VAI
\end{tabular}

\begin{tabular}{ccc}
\multicolumn{2}{c}{ Cutoff values } \\
\hline PCOS & & Normal \\
27.1 & & 27.1 \\
89.5 & & 88.5 \\
0.84 & & 0.78 \\
38 & & 36.9 \\
3.1 & 2.8 \\
\hline
\end{tabular}

\begin{tabular}{|c|c|}
\hline \multicolumn{2}{|c|}{ AUC } \\
\hline PCOS & Normal \\
\hline 0.79 & 0.74 \\
\hline 0.85 & 0.72 \\
\hline 0.67 & 0.68 \\
\hline 0.83 & 0.86 \\
\hline 0.8 & 0.89 \\
\hline
\end{tabular}

\begin{tabular}{|c|c|}
\hline \multicolumn{2}{|c|}{ SS (\%) } \\
\hline PCOS & Normal \\
\hline 0.68 & 0.78 \\
\hline 0.83 & 0.87 \\
\hline 0.73 & 0.47 \\
\hline 0.74 & 0.83 \\
\hline 0.81 & 0.88 \\
\hline
\end{tabular}

\begin{tabular}{|c|c|}
\hline \multicolumn{2}{|c|}{ SP (\%) } \\
\hline PCOS & Normal \\
\hline 0.91 & 0.69 \\
\hline 0.86 & 0.57 \\
\hline 0.61 & 0.9 \\
\hline 0.91 & 0.9 \\
\hline 0.78 & 0.9 \\
\hline
\end{tabular}

\begin{tabular}{|c|c|c|c|}
\hline \multicolumn{2}{|c|}{ PPV (\%) } & \multicolumn{2}{|c|}{ NPV (\%) } \\
\hline PCOS & Normal & PCOS & Normal \\
\hline 0.98 & 0.94 & 0.30 & 0.3 \\
\hline 0.97 & 0.93 & 0.45 & 0.39 \\
\hline 0.92 & 0.97 & 0.26 & 0.19 \\
\hline 0.98 & 0.98 & 0.36 & 0.43 \\
\hline 0.95 & 0.98 & 0.40 & 0.52 \\
\hline
\end{tabular}

PCOS, polycystic ovary syndrome; WHR, waist-to-hip ratio; WC, waist circumference; VAl, visceral adiposity index; LAP, lipid accumulation product; MetS, metabolic syndrome; AUC, area under curve; SS, sensitivity; SP, specificity; PPV, positive predictive value; NPV, negative predictive value.

the anatomic and physiological alterations related to lipid overaccumulation, and when compared with the BMI, the LAP index is more correlated with lipid risk factors (16). The VAI is also a marker of impaired fat distribution and expresses the altered function of adipocytokines, indicating a possibility of metabolic disorder $(17,52)$.

Consistent with our observations, in the study by Knowles et al. (53), the VAI and WHR were the strongest and weakest predictors of MetS in a normal population respectively. Similarly, Wiltgen et al. (2009) and Amato et al. (2013) found the LAP index and VAI as reliable and easily obtainable measures of cardiovascular risk in patients with PCOS. Furthermore, in agreement with our results, Kahn (16) reported that the LAP index value is a more reliable indicator, compared with the BMI, in prediction of metabolic disturbances. However, some reports are inconsistent with our results; for instance, Wildman et al. (54) found the WC and BMI to be the same in CVD prediction of normal subjects, or Vazquez et al. (55) reported a close ability of the BMI, WHR, and WC in the prediction of type 2 diabetes. Apart from the different diagnostic criteria used for MetS or PCOS, these discrepancies could also be attributed to genetic factors, lifestyle characteristics, and dietary habits. Another contributing factor can be the lower BMI of our subjects with PCOS in comparison with those of other studies. Finally, the method of recruitment of subjects with PCOS and control subjects can seriously influence the estimates of MetS prevalence.

Although it is confirmed that IR has a key role in the reproductive and metabolic disturbances of patients with PCOS (1), its diagnosis is still a challenge. The gold standard of the euglycemic hyperinsulinemic clamping method is expensive and time-consuming, and in the same way the indexes that depend on FPG are not easily accessible. Therefore, the markers mentioned herein may serve as easy, inexpensive, accurate, and sensitive tools in non-clinical settings.

This study has a number of strengths; the main one is a community-based sample instead of recruitment from a referral center. In addition, we used national cutoff points of the HOMA-IR for the definition of IR. However, a potential limitation that needs to be mentioned is that we used the HOMA-IR as a surrogate marker for assessing IR (6). In spite of a good correlation between the HOMA-IR and the gold standard clamp methods $(19,27)$, it might be inaccurate in PCOS (56). Furthermore, our study has a drawback that we did not have adequate power to subanalyze our data in order to compare the adiposity indexes of different phenotypes of women with PCOS.

Overall, the appropriate adiposity indicators and their optimum cutoff values vary in women with PCOS compared with the normo-ovulatory women with no androgen excess. The LAP index is an easily obtainable index that might be useful for screening of cardiometabolic complications among both these groups of women, but with different threshold levels.

Declaration of interest

The authors declare that there is no conflict of interest that could be perceived as prejudicing the impartiality of the research reported.

\section{Funding}

This research did not receive any specific grant from any funding agency in the public, commercial or not-for-profit sector.

Author contribution statement

F Ramezani Tehrani conceptualized the study and contributed to the study design, execution, analysis, and critical discussion. S Minooee contributed to the analysis and drafting of the manuscript. F Azizi contributed to the study design, execution, and drafting of the manuscript. 
13 Zimmet P, Magliano D, Matsuzawa Y, Alberti G \& Shaw J. The metabolic syndrome: a global public health problem and a new definition. Journal of Atherosclerosis and Thrombosis 200512 295-300. (doi:10.5551/jat.12.295)

\section{Acknowledgements}

The authors appreciate the substantial time and effort study participants contributed to this study. Their special thanks go to Dr Maryam Tohidi for her important contribution on laboratory testing and to Dr Homeira Rashidi for her valuable contribution in Khuzestan survey. They also wish to acknowledge Ms Niloofar Shiva for critical editing of English grammar and syntax of the manuscript. Place of study: Urban areas of Ghazvin, Kermanshah, Golestan and Hormozgan and khouzestan provinces of Iran.

\section{References}

1 Dunaif A, Graf M, Mandeli J, Laumas V \& Dobrjansky A. Characterization of groups of hyperandrogenic women with acanthosis nigricans, impaired glucose tolerance, and/or hyperinsulinemia. Journal of Clinical Endocrinology and Metabolism 198765 499-507. (doi:10.1210/jcem-65-3-499)

2 Conway GS, Agrawal R, Betteridge DJ \& Jacobs HS. Risk factors for coronary artery disease in lean and obese women with the polycystic ovary syndrome. Clinical Endocrinology 199237 119-125. (doi:10.1111/ j.1365-2265.1992.tb02295.x)

3 Wild RA, Carmina E, Diamanti-Kandarakis E, Dokras A, EscobarMorreale HF, Futterweit W, Lobo R, Norman RJ, Talbott E \& Dumesic DA. Assessment of cardiovascular risk and prevention of cardiovascular disease in women with the polycystic ovary syndrome: a consensus statement by the Androgen Excess and Polycystic Ovary Syndrome (AE-PCOS) Society. Journal of Clinical Endocrinology and Metabolism 201095 2038-2049. (doi:10.1210/jc.2009-2724)

4 Diamanti-Kandarakis E. Insulin resistance in PCOS. Endocrine 200630 13-17. (doi:10.1385/ENDO:30:1:13)

5 Duleba AJ. Medical management of metabolic dysfunction in PCOS. Steroids 201277 306-311. (doi:10.1016/j.steroids.2011.11.014)

6 Cussons AJ, Stuckey BG \& Watts GF. Metabolic syndrome and cardiometabolic risk in PCOS. Current Diabetes Reports 20077 66-73. (doi:10.1007/s11892-007-0012-8)

7 Legro RS, Arslanian SA, Ehrmann DA, Hoeger KM, Murad MH, Pasquali R \& Welt CK. Diagnosis and treatment of polycystic ovary syndrome: an endocrine society clinical practice guideline. Journal of Clinical Endocrinology and Metabolism 201398 4565-4592. (doi:10.1210/ jc.2013-2350)

8 Godinjak A, Godinjak Z, Burekovic A, Surkovic I, DizdarevicBostandzic A \& Velija-Asimi Z. Insulin resistance and lipid accumulation product in corelation to body mass index in women with polycystic ovary syndrome. Medicinski Arhiv 201266 409-411. (doi:10.5455/medarh.2012.66.409-411)

9 Amato MC, Guarnotta V, Forti D, Donatelli M, Dolcimascolo S \& Giordano C. Metabolically healthy polycystic ovary syndrome (MH-PCOS) and metabolically unhealthy polycystic ovary syndrome (MU-PCOS): a comparative analysis of four simple methods useful for metabolic assessment. Human Reproduction 201328 1919-1928. (doi:10.1093/humrep/det105)

10 Hosseinpanah F, Barzin M, Erfani H, Serahati S, Ramezani Tehrani F \& Azizi F. Lipid accumulation product and insulin resistance in Iranian PCOS prevalence study. Clinical Endocrinology 2014. In press. (doi:10.1111/cen.12287)

11 Bener A, Yousafzai MT, Darwish S, Al-Hamaq AO, Nasralla EA \& Abdul-Ghani M. Obesity index that better predict metabolic syndrome: body mass index, waist circumference, waist hip ratio, or waist height ratio. Journal of Obesity 20132013 269038. (doi:10.1155/2013/ 269038)

12 Savva SC, Lamnisos D \& Kafatos AG. Predicting cardiometabolic risk: waist-to-height ratio or BMI. A meta-analysis. Diabetes, Metabolic Syndrome and Obesity: Targets and Therapy 20136 403-419. (doi:10.2147/DMSO.S34220)
14 Dobbelsteyn CJ, Joffres MR, MacLean DR \& Flowerdew G. A comparative evaluation of waist circumference, waist-to-hip ratio and body mass index as indicators of cardiovascular risk factors. The Canadian Heart Health Surveys. International Journal of Obesity and Related Metabolic Disorders 200125 652-661. (doi:10.1038/sj.ijo. 0801582)

15 Janssen I, Katzmarzyk PT \& Ross R. Body mass index, waist circumference, and health risk: evidence in support of current National Institutes of Health guidelines. Archives of Internal Medicine 2002162 2074-2079. (doi:10.1001/archinte.162.18.2074)

16 Kahn HS. The "lipid accumulation product" performs better than the body mass index for recognizing cardiovascular risk: a populationbased comparison. BMC Cardiovascular Disorders 2005526 (doi:10.1186/1471-2261-5-26)

17 Amato MC, Giordano C, Galia M, Criscimanna A, Vitabile S, Midiri M \& Galluzzo A. Visceral adiposity index: a reliable indicator of visceral fat function associated with cardiometabolic risk. Diabetes Care 201033 920-922. (doi:10.2337/dc09-1825)

18 Hosseinpanah F, Barzin M, Tehrani FR \& Azizi F. The lack of association between polycystic ovary syndrome and metabolic syndrome: Iranian PCOS prevalence study. Clinical Endocrinology 201175 692-697. (doi:10.1111/j.1365-2265.2011.04113.x)

19 Essah PA \& Nestler JE. The metabolic syndrome in polycystic ovary syndrome. Journal of Endocrinological Investigation 200629 270-280. (doi:10.1007/BF03345554)

20 Wiltgen D, Benedetto IG, Mastella LS \& Spritzer PM. Lipid accumulation product index: a reliable marker of cardiovascular risk in polycystic ovary syndrome. Human Reproduction 200924 1726-1731. (doi:10.1093/humrep/dep072)

21 Tehrani FR, Simbar M, Tohidi M, Hosseinpanah F \& Azizi F. The prevalence of polycystic ovary syndrome in a community sample of Iranian population: Iranian PCOS prevalence study. Reproductive Biology and Endocrinology 20119 39. (doi:10.1186/1477-7827-9-39)

22 Rashidi H, Ramezani Tehrani F, Bahri Khomami M, Tohidi M \& Azizi F. To what extent does the use of the Rotterdam criteria affect the prevalence of polycystic ovary syndrome? A community-based study from the Southwest of Iran. European Journal of Obstetrics, Gynecology, and Reproductive Biology 2013174 100-105. (doi:10.1016/j. ejogrb.2013.12.018)

23 Revised 2003 consensus on diagnostic criteria and long-term health risks related to polycystic ovary syndrome (PCOS). Human Reproduction 200419 41-47. (doi:10.1093/humrep/deh098)

24 Alberti KG, Eckel RH, Grundy SM, Zimmet PZ, Cleeman JI, Donato KA, Fruchart JC, James WP, Loria CM \& Smith SC Jr. Harmonizing the metabolic syndrome: a joint interim statement of the International Diabetes Federation Task Force on Epidemiology and Prevention; National Heart, Lung, and Blood Institute; American Heart Association; World Heart Federation; International Atherosclerosis Society; and International Association for the Study of Obesity. Circulation 2009120 1640-1645. (doi:10.1161/CIRCULATIONAHA.109.192644)

25 Delavari A, Forouzanfar MH, Alikhani S, Sharifian A \& Kelishadi R. First nationwide study of the prevalence of the metabolic syndrome and optimal cutoff points of waist circumference in the Middle East: the national survey of risk factors for noncommunicable diseases of Iran. Diabetes Care 200932 1092-1097. (doi:10.2337/dc08-1800)

26 Tehrani FR, Rashidi H \& Azizi F. The prevalence of idiopathic hirsutism and polycystic ovary syndrome in the Tehran Lipid and Glucose Study. Reproductive Biology and Endocrinology 20119144. (doi:10.1186/1477-7827-9-144)

27 Wallace TM, Levy JC \& Matthews DR. Use and abuse of HOMA modeling. Diabetes Care 200427 1487-1495. (doi:10.2337/ diacare.27.6.1487) 
Gynecology, and Reproductive Biology 2005122 206-212. (doi:10.1016/j. ejogrb.2005.03.010)

28 Zadeh-Vakili A, Tehrani FR \& Hosseinpanah F. Waist circumference and insulin resistance: a community based cross sectional study on reproductive aged Iranian women. Diabetology \& Metabolic Syndrome 20113 18. (doi:10.1186/1758-5996-3-18)

29 Akobeng AK. Understanding diagnostic tests 3: receiver operating characteristic curves. Acta Paediatrica 200796 644-647. (doi:10.1111/ j.1651-2227.2006.00178.x)

30 Youden WJ. Index for rating diagnostic tests. Cancer 19503 32-35. (doi:10.1002/1097-0142(1950)3:1 <32::AID-CNCR2820030106>3.0. $\mathrm{CO} ; 2-3)$

31 Flegal KM, Carroll MD, Ogden CL \& Curtin LR. Prevalence and trends in obesity among US adults, 1999-2008. Journal of the American Medical Association 2010303 235-241. (doi:10.1001/jama.2009.2014)

32 Lim SS, Davies MJ, Norman RJ \& Moran LJ. Overweight, obesity and central obesity in women with polycystic ovary syndrome: a systematic review and meta-analysis. Human Reproduction Update 201218 618-637. (doi:10.1093/humupd/dms030)

33 Svendsen PF, Nilas L, Norgaard K, Jensen JE \& Madsbad S. Obesity, body composition and metabolic disturbances in polycystic ovary syndrome. Human Reproduction 200823 2113-2121. (doi:10.1093/humrep/den211)

34 Cosar E, Ucok K, Akgun L, Koken G, Sahin FK, Arioz DT \& Bas O. Body fat composition and distribution in women with polycystic ovary syndrome. Gynecological Endocrinology 200824 428-432. (doi:10.1080/ 09513590802234253)

35 Godoy-Matos AF, Vaisman F, Pedrosa AP, Farias ML, Mendonca LM \& Pinheiro MF. Central-to-peripheral fat ratio, but not peripheral body fat, is related to insulin resistance and androgen markers in polycystic ovary syndrome. Gynecological Endocrinology 200925 793-798. (doi:10.3109/09513590903015528)

36 Karabulut A, Yaylali GF, Demirlenk S, Sevket O \& Acun A. Evaluation of body fat distribution in PCOS and its association with carotid atherosclerosis and insulin resistance. Gynecological Endocrinology 2012 28 111-114. (doi:10.3109/09513590.2011.589929)

37 Yucel A, Noyan V \& Sagsoz N. The association of serum androgens and insulin resistance with fat distribution in polycystic ovary syndrome. European Journal of Obstetrics, Gynecology, and Reproductive Biology 2006 126 81-86. (doi:10.1016/j.ejogrb.2005.11.012)

38 Carmina E, Bucchieri S, Esposito A, Del Puente A, Mansueto P, Orio F, Di Fede G \& Rini G. Abdominal fat quantity and distribution in women with polycystic ovary syndrome and extent of its relation to insulin resistance. Journal of Clinical Endocrinology and Metabolism 200792 2500-2505. (doi:10.1210/jc.2006-2725)

39 Lee H, Oh JY, Sung YA \& Chung H. Is insulin resistance an intrinsic defect in Asian polycystic ovary syndrome? Yonsei Medical Journal 2013 54 609-614. (doi:10.3349/ymj.2013.54.3.609)

40 Ketel IJ, Serne EH, Ijzerman RG, Korsen TJ, Twisk JW, Hompes PG, Smulders YM, Homburg R, Vorstermans L, Stehouwer CD et al. Insulin-induced capillary recruitment is impaired in both lean and obese women with PCOS. Human Reproduction 201126 3130-3137. (doi:10.1093/humrep/der296)

41 Vrbikova J, Cibula D, Dvorakova K, Stanicka S, Sindelka G, Hill M, Fanta M, Vondra K \& Skrha J. Insulin sensitivity in women with polycystic ovary syndrome. Journal of Clinical Endocrinology and Metabolism 200489 2942-2945. (doi:10.1210/jc.2003-031378)

42 Glueck CJ, Dharashivkar S, Wang P, Zhu B, Gartside PS, Tracy T \& Sieve L. Obesity and extreme obesity, manifest by ages $20-24$ years, continuing through 32-41 years in women, should alert physicians to the diagnostic likelihood of polycystic ovary syndrome as a reversible underlying endocrinopathy. European Journal of Obstetrics,
43 Hahn S, Tan S, Sack S, Kimmig R, Quadbeck B, Mann K \& Janssen OE. Prevalence of the metabolic syndrome in German women with polycystic ovary syndrome. Experimental and Clinical Endocrinology \& Diabetes 2007115 130-135. (doi:10.1055/s-2007-967093)

44 Vrbikova J, Dvorakova K, Grimmichova T, Hill M, Stanicka S, Cibula D, Bendlova B, Starka L \& Vondra K. Prevalence of insulin resistance and prediction of glucose intolerance and type 2 diabetes mellitus in women with polycystic ovary syndrome. Clinical Chemistry and Laboratory Medicine 200745 639-644. (doi:10.1515/CCLM.2007.113)

45 Mukherjee S, Shaikh N, Khavale S, Shinde G, Meherji P, Shah N \& Maitra A. Genetic variation in exon 17 of INSR is associated with insulin resistance and hyperandrogenemia among lean Indian women with polycystic ovary syndrome. European Journal of Endocrinology 2009160 855-862. (doi:10.1530/EJE-08-0932)

46 Wang K, Wang L, Zhao Y, Shi Y, Wang L \& Chen ZJ. No association of the Arg51Gln and Leu72Met polymorphisms of the ghrelin gene and polycystic ovary syndrome. Human Reproduction 200924 485-490. (doi:10.1093/humrep/den430)

47 Panidis D, Tziomalos K, Chatzis P, Papadakis E, Delkos D, Tsourdi EA, Kandaraki EA \& Katsikis I. Association between menstrual cycle irregularities and endocrine and metabolic characteristics of the polycystic ovary syndrome. European Journal of Endocrinology 2013168 145-152. (doi:10.1530/EJE-12-0655)

48 Goodarzi MO, Dumesic DA, Chazenbalk G \& Azziz R. Polycystic ovary syndrome: etiology, pathogenesis and diagnosis. Nature Reviews. Endocrinology 20117 219-231. (doi:10.1038/nrendo.2010.217)

49 DeUgarte CM, Bartolucci AA \& Azziz R. Prevalence of insulin resistance in the polycystic ovary syndrome using the homeostasis model assessment. Fertility and Sterility 200583 1454-1460. (doi:10.1016/ j.fertnstert.2004.11.070)

50 Mukherjee S \& Maitra A. Molecular \& genetic factors contributing to insulin resistance in polycystic ovary syndrome. Indian Journal of Medical Research 2010131 743-760.

51 Diamanti-Kandarakis E \& Dunaif A. Insulin resistance and the polycystic ovary syndrome revisited: an update on mechanisms and implications. Endocrine Reviews 201233 981-1030. (doi:10.1210/er.2011-1034)

52 Amato MC, Verghi M, Galluzzo A \& Giordano C. The oligomenorrhoic phenotypes of polycystic ovary syndrome are characterized by a high visceral adiposity index: a likely condition of cardiometabolic risk. Human Reproduction 201126 1486-1494. (doi:10.1093/humrep/der088)

53 Knowles KM, Paiva LL, Sanchez SE, Revilla L, Lopez T, Yasuda MB, Yanez ND, Gelaye B \& Williams MA. Waist circumference, body mass index, and other measures of adiposity in predicting cardiovascular disease risk factors among peruvian adults. International Journal of Hypertension 20112011 931402. (doi:10.4061/2011/931402)

54 Wildman RP, Gu D, Reynolds K, Duan X, Wu X \& He J. Are waist circumference and body mass index independently associated with cardiovascular disease risk in Chinese adults? American Journal of Clinical Nutrition 200582 1195-1202.

55 Vazquez G, Duval S, Jacobs DR Jr \& Silventoinen K. Comparison of body mass index, waist circumference, and waist/hip ratio in predicting incident diabetes: a meta-analysis. Epidemiologic Reviews 200729 115-128. (doi:10.1093/epirev/mxm008)

56 Costa EC, Sa JC, Soares EM, Lemos TM, Maranhao TM \& Azevedo GD. Anthropometric indices of central obesity how discriminators of metabolic syndrome in Brazilian women with polycystic ovary syndrome. Gynecological Endocrinology 201228 12-15. (doi:10.3109/ 09513590.2011.583956)

Received 3 February 2014

Revised version received 8 April 2014

Accepted 9 May 2014 